\title{
Frontal Fibrosing Alopecia and Autoimmune Disorders in a Hispanic Female
}

\author{
Jessica Carolina Martinez-Rico Jesus Ancer-Arellano Adrian Martinez-Moreno \\ Sonia Sofia Ocampo-Garza Jorge Ocampo-Candiani Sonia Chavez-Alvarez \\ Department of Dermatology, Universidad Autonoma de Nuevo Leon, University Hospital \\ "Dr. José Eleuterio González," Monterrey, Mexico
}

\section{Established Facts}

- Autoimmune diseases have an unclear etiology and complex diagnosis, and their treatment is challenging.

- The etiology of frontal fibrosing alopecia remains unclear; nonetheless, its association with diseases such as vitiligo and thyroid disorders suggests an underlying autoimmune mechanism.

\section{Novel Insights}

- Understanding the phenomena of co-localization in autoimmune cutaneous diseases alongside frontal fibrosing alopecia still needs further research.

- JAK/STAT inhibitors target a common immunological pathway and might be a potentially effective treatment for patients with multiple autoinflammatory skin diseases.

\section{Keywords}

Frontal fibrosing alopecia - Vitiligo - Autoimmune disease · Hypopigmentation · Dyschromia

\section{Abstract \\ Frontal fibrosing alopecia (FFA) is a cicatricial alopecia char- acterized by hairline recession. Multiple autoimmune pa- thologies have been reported in patients with FFA. Despite the fact that FFA etiology remains unknown, there has been described an association with autoimmune disorders prob-}

ably caused by an altered activity of cytotoxic CD8 T lymphocytes. Moreover, other autoimmune pathologies develop $\mathrm{TH} 1$ and $\mathrm{TH} 17$ response. Genetics could be responsible, in part, for the role of multiple simultaneous autoimmune disorders. Herein, we describe a case of a female patient with vitiligo, lichen sclerosus, and autoimmune hypothyroidism who developed a pruritic band-like recession of the frontal hairline. More research is needed in this area since autoimmune events in these patients may not be a mere coincidence.

(c) 2020 S. Karger AG, Basel

$\begin{aligned} & \text { karger@karger.com } \\ & \text { www.karger.com/sad }\end{aligned}$
Karger ${ }^{\prime /}$




\section{Introduction}

Autoimmune pathologies have become more prevalent in the general population. Additionally, a better comprehension of the physiological and immunological pathways has improved the diagnosis and treatment for patients. The coexistence of multiple autoinflammatory skin diseases in frontal fibrosing alopecia (FFA) is scarcely mentioned. Herein, we present a case with multiple autoinflammatory manifestations (vitiligo, lichen sclerosus, and autoimmune hypothyroidism) besides FFA.

\section{Case Report/Case Presentation}

A 76-year-old postmenopausal Hispanic female with a personal history of diabetes mellitus type 2, hypothyroidism, vitiligo, and genital lichen sclerosus presented to the outpatient clinic complaining of a band-like recession of the frontal hairline. She mentioned hair loss accompanied by moderate pruritus began 5 years prior (Fig. 1).

Upon physical examination, she had eyebrow loss and the coexistence of an achromic patch along the scalp, making the hairline recession more evident. Disseminated achromic patches were also seen in other areas of her body. The patient mentioned that the macules preceded the hair loss. These macules were fluorescent when examined with Wood's lamp, making them compatible with vitiligo. Dermoscopy showed lonely hairs, perifollicular erythema, scaling, and loss of follicular openings (Fig. 1b). A diagnosis of FFA coexistent with vitiligo was made. Dermoscopy-guided punch biopsies were performed confirming both vitiligo (negative MelanA, S-100, and HMB-45) and FFA (Fig. 1c). In the genital area, she had a 5-year history of lichen sclerosus, for which she had not received diagnosis nor treatment.

\section{Discussion/Conclusion}

Autoimmune diseases have an unclear etiology, diagnosing them is relatively complex, and their treatment is challenging. New research has explained multiple inflammatory pathways of autoimmune diseases and, therefore, improved the therapeutic approach.

FFA is a lymphocytic cicatricial alopecia that occurs mainly in postmenopausal women. Etiology remains unclear; however, its association with vitiligo and thyroid disorders suggests an underlying autoimmune mechanism $[1,2]$.

It is believed that coexistence of vitiligo in affected areas by FFA is not a coincidence but a manifestation of an altered activity of cytotoxic CD8 T lymphocytes. This damages basal keratinocytes and the external hair root sheath. This leads to apoptosis with consequent depigmentation
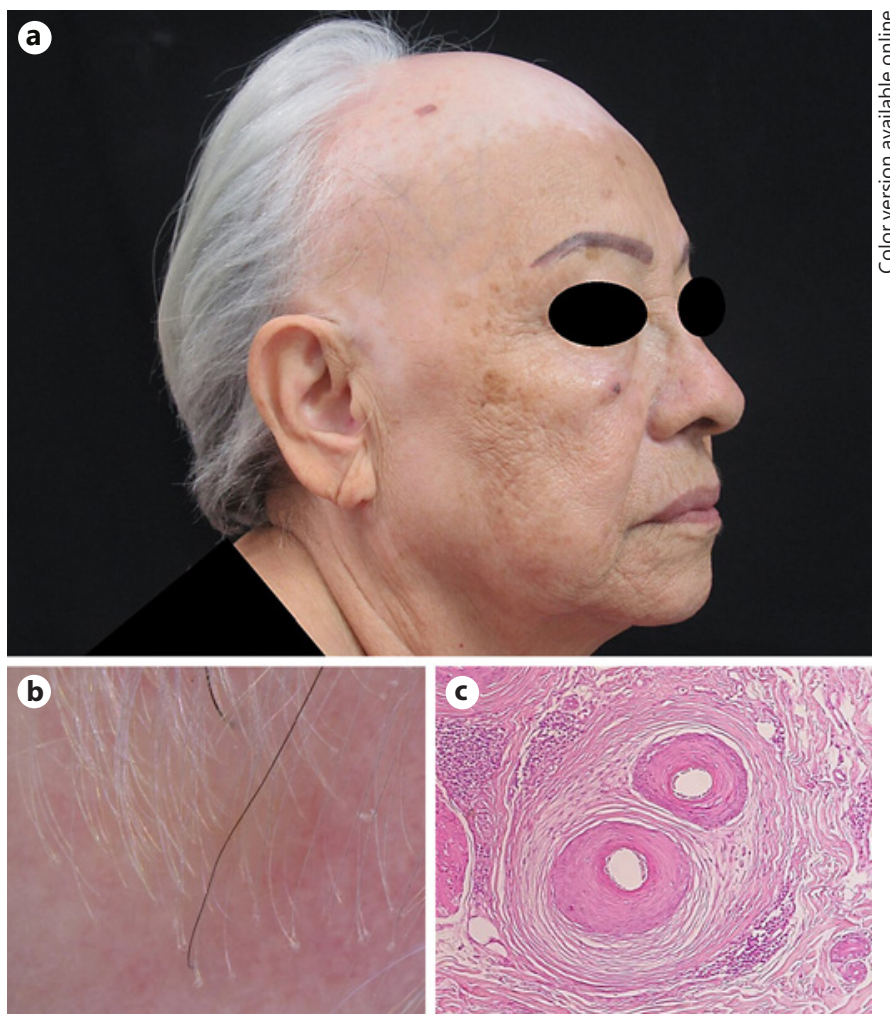

Fig. 1 a Seventy-six-year-old patient with coexistence of FFA and vitiligo. b Dermoscopy. Perifollicular erythema and hyperkeratosis and loss of follicular openings. c Histology. Perifollicular fibrosis and lymphocytic lichenoid infiltrate. Hematoxylin and eosin stain. Original magnification $\times 400$. FFA, frontal fibrosing alopecia.

and irreversible destruction of the hair follicle [3]. Plasmacytoid dendritic cells intervene as the main source of interferon 1, which plays an important role in the migration of cytotoxic $\mathrm{T}$ cells orchestrating this cellular damage. This pathway has been shown to be harmful for other conditions like vitiligo, lichen planus, lichen sclerosus et atrophicus, and lupus erythematosus. This damage mostly occurs in perilesional vitiligo skin, showing its participation in early developing lesions and lately in some hair diseases [4-7].

Hair follicle is an immune-privileged (IP) anatomical site, protected against autoimmune injury. When AA occurs, a loss in this IP causes an ectopic expression of class I major histocompatibility complex, leading to a CD8 Tlymphocyte-derived inflammatory process. It is known that lichen planopilaris and FFA are brought on by this phenomenon. An IFN $\gamma$-induced IP loss happens due to a reduction of TFG- $\beta 2$ and CD200 and overexpression of major histocompatibility complex class I, II, and beta-2 microglobulin, similar to the autoimmune pathophysiology of 
Table 1. Coexistence of FFA, vitiligo, and lichen sclerosus et atrophicus in reported cases

\begin{tabular}{ccclll}
\hline No. & Sex & $\begin{array}{c}\text { Age, } \\
\text { years }\end{array}$ & Autoimmune manifestation & Race & Reference \\
\hline 1 & F & 55 & Co-localized vitiligo & Caucasian & Miteva et al. [3] \\
2 & $F^{*}$ & 67 & Co-localized vitiligo and hypothyroidism & Caucasian & Miteva et al. [3] \\
3 & $F^{*}$ & 67 & Co-localized vitiligo and hypothyroidism & Caucasian & Miteva et al. [3] \\
4 & F & 42 & Co-localized vitiligo & Hispanic & Miteva et al. [3] \\
5 & F & 61 & Co-localized vitiligo & Caucasian & Vañó-Galván et al. [1] \\
6 & F & 77 & Co-localized vitiligo & Caucasian & Vañó-Galván et al. [1] \\
7 & F & 72 & Co-localized vitiligo, autoimmune thyroid disease, and morphea & Caucasian & Katoulis et al. [11] \\
8 & M & 48 & Co-localized vitiligo & Caucasian & Katoulis et al. [11] \\
9 & F & 60 & Lichen sclerosus & Caucasian & Feldmann et al. [23] \\
$10-13$ & - & - & Lichen sclerosus & - & Strazzulla et al. [17] \\
14 & F & - & Lichen sclerosus & Caucasian & Vañó-Galván et al. [1] \\
15 & F & - & Genital lichen sclerosus & Caucasian & MacDonald et al. [24] \\
16 & F & - & Genital lichen sclerosus et al. [16] \\
17 & F & 76 & Diabetes mellitus, hypothyroidism, and genital lichen sclerosus & Hispanic & This study \\
\hline
\end{tabular}

FFA, frontal fibrosing alopecia; F, female; M, male. * Patients were identical twin sisters.

alopecia areata [8]. FFA has been also associated with other autoimmune diseases. The coexistence of discoid lupus erythematosus (DLE) and FFA has been published. One case reported by Gaffney et al. presented a patient with DLE who 2 years later developed alopecia consistent with FFA. Moreover, Khan et al. described a patient with FFA and photosensitivity, with histopathology compatible with FFA but direct immunofluorescence consistent with DLE. Autoimmunity may be a triggering factor associating two or more autoimmune diseases. Genetic predisposition may also play a role in concomitant appearance $[4,9]$.

Lichen sclerosus may also occur simultaneously with other autoimmune diseases, like vitiligo [10]. Chronic inflammation seen in these entities could explain the coexistence of diseases in the same location. Nevertheless, more studies are needed to validate this hypothesis. Another proposed mechanism is the Koebner phenomenon, associated to cellular inflammation and injury, like the one previously described [11]. Garcia-Souto et al. [12] recently described a patient with a history of vitiligo and psoriasis who developed lesions consistent with FFA where preexisting vitiligo was located. In our patient, a Koebner phenomenon secondary to photodamage could explain the three coexisting entities, caused by a T-cell lymphocytic inflammatory process [12].

Morphea (specifically coup de sabre or linear morphea) has been recently associated to FFA. These two diseases share a common inflammatory signaling process which induces polarization toward $\mathrm{TH} 1$ and $\mathrm{TH} 17$ immune response. Additionally, in scleroderma, fibrosing alopecia is due to an elevation of these lymphocytes, whereas cytokines derived from $\mathrm{TH} 2$ are related to late immune fibrosis in its late stage [13]. Other connective tissue and autoimmune disorders have also been reported in patients with FFA. Sjögren's disease with FFA has occurred in a Japanese and a Brazilian woman [14].

In the largest case series of FFA published in the literature, we have found that the most prevalent autoimmune diseases are thyroid disorders with percentages ranging from 7.6 to $37.1 \%$ (with hypothyroidism being the most frequent), alopecia areata $0.4-13 \%$, vitiligo $0.5-7.7 \%$, lichen sclerosus $0.2-4 \%$, psoriasis $2-3 \%$, and cutaneous lupus erythematosus $1.1-3 \%[1,15-19]$. There are also some other coexistent entities reported less frequently. Some examples of these are primary biliary cirrhosis, rheumatoid arthritis, systemic lupus erythematosus, polymyositis, polymyalgia rheumatica, systemic and localized sclerosis, Sjögren's syndrome, autoimmune hepatitis, giant cell arteritis, celiac disease, and inflammatory intestinal diseases $[14,19,20]$.

Vitiligo and FFA have also been observed. Most cases involve vitiligo as an initial cutaneous manifestation and later, FFA (Table 1). Hypopigmentation on the scalp of these patients in similar clinical scenarios has been reported, finding decreased epidermal melanocyte count when compared to patients with unaffected scalp. The decrease in melanocytes, evidenced by pathology, underlines the importance of the immunopathogenic origin, and its consequences are represented as pigment alterations and alopecia [21]. 
FFA coexisting with other autoimmune cutaneous diseases has been seldomly described. The association between multiple inflammatory diseases, such as vitiligo, lichen sclerosus, and FFA, remains poorly understood. It is quite uncommon that they present simultaneously; however, this might be possible due to the similarity in their immune signaling pathway. Treatment for these conditions is challenging. A medication that targets a common immunological pathway (like JAK/STAT inhibitors) might be a potentially effective treatment for patients with multiple autoinflammatory skin diseases [22]. Nonetheless, more studies with a large quantity of patients and a longer follow-up period are required to reach conclusions.

\section{Statement of Ethics}

Written informed consent was obtained from the patient for publication.

\section{Conflict of Interest Statement}

The authors have no conflicts of interest to declare.

\section{Funding Sources}

The study was funded by own resources.

\section{Author Contributions}

J.C.M.R. contributed to the design, drafting, intellectual content, final approval, and agreement of this work. J.A.A. contributed to the design, drafting, intellectual content, final approval, and agreement of this work. A.M.M. contributed to the design, drafting, intellectual content, final approval, and agreement of this work. S.S.O.G. contributed to the design, drafting, intellectual content, final approval, and agreement of this work. J.O.C. contributed to the design, drafting, intellectual content, final approval, and agreement of this work. S.C.A. contributed to the design, drafting, intellectual content, final approval, and agreement of this work.

\section{References}

1 Vañó-Galván S, Molina-Ruiz AM, SerranoFalcón C, Arias-Santiago S, Rodrigues-Barata AR, Garnacho-Saucedo G, et al. Frontal fibrosing alopecia: a multicenter review of 355 patients. J Am Acad Dermatol. 2014 Apr;70(4): $670-8$.

2 To D, Beecker J. Frontal fibrosing alopecia: update and review of challenges and successes. J Cutan Med Surg. 2018 Mar/Apr;22(2):182-9.

3 Miteva M, Aber C, Torres F, Tosti A. Frontal fibrosing alopecia occurring on scalp vitiligo: report of four cases. Br J Dermatol. 2011 Aug; 165(2):445-7.

4 del Rei M, Pirmez R, Sodré CT, Tosti A. Coexistence of frontal fibrosing alopecia and discoid lupus erythematosus of the scalp in $7 \mathrm{pa}$ tients: just a coincidence? J Eur Acad Dermatol Venereol. 2016 Jan;30(1):151-3.

5 Wenzel J, Wiechert A, Merkel C, Bieber T, Tüting T. IP10/CXCL10-CXCR3 interaction: a potential self-recruiting mechanism for cytotoxic lymphocytes in lichen sclerosus et atrophicus. Acta Derm Venereol. 2007;87(2):112-

6 Bertolotti A, Boniface K, Vergier B, Mossalayi D, Taieb A, Ezzedine K, et al. Type I interferon signature in the initiation of the immune response in vitiligo. Pigment Cell Melanoma Res. 2014 May;27(3):398-407.

7 Abou Rahal J, Kurban M, Kibbi AG, Abbas O. Plasmacytoid dendritic cells in alopecia areata: missing link? J Eur Acad Dermatol Venereol. 2016 Jan;30(1):119-23.

8 Tziotzios C, Stefanato CM, Fenton DA, Simpson MA, McGrath JA. Frontal fibrosing alopecia: reflections and hypotheses on aetiology and pathogenesis. Exp Dermatol. 2016 Nov; 25(11):847-52.
9 Furlan KC, Kakizaki P, Chartuni JC, Valente NY. Frontal fibrosing alopecia in association with Sjögren's syndrome: more than a simple coincidence. An Bras Dermatol. 2016 Sep-Oct; 91(5 Suppl 1):14-6.

10 Kwon IH, Kye H, Seo SH, Ahn HH, Kye YC, Choi JE. Synchronous onset of symmetrically associated extragenital lichen sclerosus and vitiligo on both breasts and the vulva. Ann Dermatol. 2015 Aug;27(4):456-7.

11 Katoulis AC, Diamanti K, Sgouros D, Liakou AI, Alevizou A, Bozi E, et al. Frontal fibrosing alopecia and vitiligo: coexistence or true association? Skin Appendage Disord. 2017 Jan; 2(3-4):152-5.

12 Garcia-Souto F, Cases-Merida S, Sosa-Moreno F, Escudero-Ordoñez J. A singular association of vitiligo, psoriasis and frontal fibrosing alopecia. Australas J Dermatol. 2020 Feb;61(1): e127-8.

13 Abdalla BMZ, Matsunaga N, Landman G, Machado Filho CD, Criado PR. Association between frontal fibrosing alopecia and linear scleroderma "coup de sabre". Australas J Dermatol. 2019 Aug;60(3):e256-8.

14 Zhang M, Zhang L, Rosman IS, Mann CM. Frontal fibrosing alopecia demographics: a survey of 29 patients. Cutis. 2019 Feb;103(2): E16-22.

15 Kanti V, Constantinou A, Reygagne P, Vogt A, Kottner J, Blume-Peytavi U. Frontal fibrosing alopecia: demographic and clinical characteristics of 490 cases. J Eur Acad Dermatol Venereol. 2019 Oct;33(10):1976-83.

16 Valesky EM, Maier MD, Kippenberger S, Kaufmann R, Meissner M. Frontal fibrosing alopecia: review of recent case reports and case series in Pubmed. J Dtsch Dermatol Ges. 2018 Aug;16(8):992-9.
17 Strazzulla LC, Avila L, Li X, Lo Sicco K, Shapiro J. Prognosis, treatment, and disease outcomes in frontal fibrosing alopecia: a retrospective review of 92 cases. J Am Acad Dermatol. 2018 Jan;78(1):203-5.

18 Starace M, Brandi N, Alessandrini A, Bruni F, Piraccini BM. Frontal fibrosing alopecia: a case series of 65 patients seen in a single Italian centre. J Eur Acad Dermatol Venereol. 2019 Feb; 33(2):433-8.

19 Banka N, Mubki T, Bunagan MJ, McElwee K, Shapiro J. Frontal fibrosing alopecia: a retrospective clinical review of 62 patients with treatment outcome and long-term follow-up. Int J Dermatol. 2014 Nov;53(11):1324-30.

20 Eginli AN, Bagayoko CW, McMichael AJ. A case of frontal fibrosing alopecia in a patient with primary biliary cirrhosis and polymyalgia rheumatica. Skin Appendage Disord. 2016 Sep;2(1-2):79-82.

21 Lin J, Valdebran M, Bergfeld W, Conic RZ, Piliang M, Atanaskova Mesinkovska N. Hypopigmentation in frontal fibrosing alopecia. J Am Acad Dermatol. 2017 Jun;76(6):1184-6.

22 Samadi A, Ahmad Nasrollahi S, Hashemi A, Nassiri Kashani M, Firooz A. Janus kinase (JAK) inhibitors for the treatment of skin and hair disorders: a review of literature. J Dermatolog Treat. 2017 Sep;28(6):476-83.

23 Feldmann R, Harms M, Saurat J. Postmenopausal frontal fibrosing alopecia [in German]. Hautarzt. 1996;47(7):533-6.

24 MacDonald A, Clark C, Holmes S. Frontal fibrosing alopecia: a review of 60 cases. J Am Acad Dermatol. 2012;67(5):955-61.
Frontal Fibrosing Alopecia and Autoimmune Disorders
Skin Appendage Disord 2020;6:362-365 DOI: $10.1159 / 000507557$ 